\title{
Phénologie du couvert arborescent dans une chênaie-hêtraie d'Aquitaine
}

\author{
B. COMPS, Josette LETOUZEY et J.-M. SAVOIE \\ Université de Bordeaux I, Laboratoire de Botanique \\ Avenue des Facultés, F 33405 Talence
}

\begin{abstract}
Résumé
L'étude de la phénologie du couvert arborescent d'une chênaie-hêtraie d'Aquitaine a permis de décrire le comportement saisonnier du Hêtre au point de vue débourrement, défoliation et durée de la période végétative, près de la limite climatique méridionale de son aire. Des observations analogues sur le Chêne pédonculé ont permis de comparer la phénologie des deux espèces, d'une part entre elles en région Aquitaine, d'autre part avec celle des peuplements localisés plus au nord dans l'aire optimale du Hêtre.

En Aquitaine, l'estimation de la date de débourrement par les méthodes de somme de températures peut s'effectuer en choisissant le début avril comme date d'initialisation ; les températures plus élevées du printemps à cette latitude favorisent la précocité et la vitesse de foliation. Une discussion est amorcée pour tenter d'évaluer le poids relatif de la photopériode et de la température sur le déterminisme de la date de foliation.

La précocité du débourrement et celle de la défoliation chez le Hêtre sont des caractères liés à l'individu donc dépendant fortement a priori de son génotype.

La foliation du Chêne pédonculé paraît plus sensible aux actions de la température qu'à la photopériode ; il est possible d'estimer la date de débourrement de cette espèce en cumulant des actions thermiques journalières à partir du $1^{\text {er }}$ janvier.

Contrairement à ce qui est couramment observé dans les régions plus septentrionales, le Hêtre entre en végétation plus tard que le Chêne en Aquitaine.
\end{abstract} Aquitaine

Mots clés : Débourrement, période végétative, somme de températures, Hêtre, Chêne pédonculé,

\section{Introduction}

Dans la partie la plus méridionale du domaine atlantique, la hêtraie climacique caractérise essentiellement l'étage montagnard humide du Massif Central, des Pyrénées et de la Cordillère cantabrique. Le secteur aquitanien, d'altitude moyenne peu élevée représente, dans l'aire du Hêtre, une limite climatique concrétisée par la dispersion et la faible superficie des peuplements de l'espèce au sein des chênaies atlantiques et subméditerranéennes.

Les observations phénologiques réalisées dans une chênaie-hêtraie d'Aquitaine (forêt de Laveyron, Landes) ont pour but principal de connaître le comportement 
saisonnier du Hêtre sur cette limite. Elles permettent également de comparer ce comportement à celui du Chêne pédonculé auquel le Hêtre est le plus souvent subordonné sur sols acides. Enfin, elles viennent compléter celles de divers auteurs dont les travaux se rapportent, pour la plupart, à l'aire optimale des hêtraies.

Le débourrement du Hêtre, en particulier, a fait l'objet depuis longtemps de nombreuses observations: influence de la latitude, de l'altitude, du type phyto-écologique de hêtraie sur la précocité du phénomène (ENgler, 1911 ; Baumgartner, KLeinLein \& WaldmanN, 1956 ; Lausi \& PignatTı, 1973...) ; influence de la température et de la photopériode (Helms, 1918 ; Wareing, 1953; Schaffalitsky de Muckadeli, 1959 ; Lavarenne-Allary, 1965 ; Galoux, 1966); mise en évidence de la stabilité relative de l'ordre d'entrée en végétation des individus composant un peuplement (Leibundgut \& Kunz, 1952 ; Schaffalitzky de Muckadell, 1959 ; Galoux, 1966...). Une synthèse des principaux résultats concernant ce secteur de la biologie du Hêtre a récemment été réalisée par BECKER (1981) et RIEDACKER (1981). Les recherches sur la coloration automnale des feuilles et la défoliation sont peu nombreuses car l'évaluation quantitative de ces phénomènes s'avère difficile (KraHL-Urban, 1958; LavarenneAllary, 1965 ; Galoux et al., 1967...).

La présente étude rapporte et commente les principaux résultats obtenus de 1973 à 1980 sur la foliation du Hêtre (comparée à celle du Chêne pédonculé), sur sa défoliation et la durée de sa période végétative en forêt de Laveyron.

\section{Méthodologie}

\subsection{Cadre d'étude}

La forêt de Laveyron (") est située sur la commune de Lussagnet, à $26 \mathrm{~km}$ au sudest de Mont-de-Marsan (Landes, 44'N) Elle recouvre le rebord et le versant à l'exposition nord d'un plateau entre 140 et $97 \mathrm{~m}$ d'altitude.

Le climat local est de type atlantique méridional, à étés relativement chauds (tm juillet $\left.=20^{\circ}\right)$ mais sans période sèche, à hivers doux ( $\mathrm{tm}$ janvier $\left.=6^{\circ} 3\right)$. La température annuelle moyenne est de $12^{\circ} 6$ et les précipitations annuelles atteignent $934 \mathrm{~mm}{ }^{(*)}$.

Les peuplements de Hêtre (rebord du plateau et versant nord) appartiennent à l'Androsaemo-Fagetum aquitain des sols acides décrit en 1980 par B. Comps et al. Selon la topographie, trois types de futaies peuvent être distingués:

- la chênaie à Chêne pédonculé (Quercus pedunculata) avec en mélange le Hêtre (Fagus silvatica), le Chêne sessile ( $Q$. sessiliflora) et le Chêne pubescent ( $Q$. pubescens avec localement $Q$. toza). Elle occupe la partie supérieure du plateau. Les arbres y sont de mauvaise venue, branchus et de faible hauteur;

- la chênaie-hêtraie à Chêne sessile et Chêne pédonculé où le Hêtre devient parfois dominant (Androsaemo-Fagetum); elle se développe sur la plus grande partie des versants ;

(*) Forêt domaniale gérée par l'O.N.F. de Mont-de-Marsan

${ }^{* *}$ ) Données météorologiques de la station de la Mćtéorologie nationale de Lussagnet distante de $1,5 \mathrm{~km}$ de la forêt de Laveyron. 
- la chênaie pédonculée à Charme (Carpinus betulus) et à Aulne (Alnus glutinosa) ; elle apparaît en bas de pente, en bordure du ruisseau. Le Hêtre, le plus souvent absent, n'apparaît que comme essence secondaire.

Trois peuplements ont été choisis, localisés dans deux situations écologiques différentes :

- 2 peuplements mixtes : peuplements fermés de versant, l'un exposé au nord-est (parcelle 9), l'autre à l'est (parcelle 25), sur pente moyenne de $7 \%$, avec $60 \%$ de hêtres et $40 \%$ de chênes ( $Q$. pedunculata et $Q$. sessiliflora). Il s'agit de futaies d'environ 100 ans, à sous-étage assez bien développé, sur sols lessivés acides, limoneux, à nappe perchée temporaire (pseudogley plus ou moins profond) ;

- peuplement de Chêne pédonculé : peuplement fermé de fond de vallée à pente nulle, sur sol alluvial hydromorphe à gley, limoneux, à mull eutrophe. La futaie de 150 ans environ domine un taillis d'Aulne et une strate arbustive à fort recouvrement (parcelle 20).

Les températures moyennes mensuelles $(1,50 \mathrm{~m}$ sous abri) calculées pour la parcelle 9 de versant et la parcelle 20 de fond de vallée (seules parcelles pour lesquelles nous disposons de données complètes pour la période 1973-1978) sont reportées sur le tableau 1. Le fond de vallée reste constamment plus froid que le versant nord, aussi bien en phase feuillée que défeuillée, surtout en raison de températures maximales plus basses, les valeurs des minimales étant assez comparables pour les deux situations topographiques.

TABLEAU 1

Températures moyennes annuelles (parcelles 9 versant et 20) pour la période 1973-1978.

\begin{tabular}{c|c|c|c|c|c|c|c|c|c|c|c|c}
\hline Parcelles & J & F & M & A & M & J & Jt & A & S & O & N & D \\
\hline 9 & 5,6 & 7,2 & 8,8 & 10,0 & 13,7 & 16,9 & 18,7 & 18,9 & 16,0 & 11,3 & 7,4 & 5,8 \\
20 & 4,8 & 6,2 & 8,1 & 9,6 & 13,4 & 16,4 & 18,2 & 18,2 & 15,4 & 10,8 & 6,8 & 5,1 \\
\hline
\end{tabular}

\subsection{La foliation}

\subsection{Méthodes d'observation}

L'apparition et le développement du feuillage, depuis le bourgeon dormant jusqu'à la feuille adulte, s'effectuent selon un processus caractéristique de l'espèce végétale. Le débourrement correspond au stade où les bourgeons, éclatés, laissent entrevoir à leur sommet les jeunes feuilles encore plissées (stade 4 de Malaisse, 1964). Les observations hebdomadaires effectuées de 1973 à 1980 consistent à apprécier globalement, sur $500 \mathrm{~m}^{2}$ environ, le pourcentage d'arbres ayant atteint le stade 4 de foliation. Une échelle analogue a été utilisée pour l'étude de la foliation du Chêne.

Des observations plus rigoureuses faisant référence aux travaux de MALAISSE (1964) viennent compléter pour le Hêtre, en 1979 et 1980, les résultats obtenus auparavant. La méthode consiste à étudier le déroulement complet du phénomène $(7$ stades caractéristi- 
ques successifs) et tient compte de la variabilité entre les individus d'un même peuplement.

L'appréciation de chaque stade s'effectue arbre par arbre pour l'ensemble de la couronne. La moyenne des observations individuelles permet ensuite d'attribuer au peuplement étudié un stade moyen de foliation.

On définit la date de débourrement comme étant celle à laquelle $50 \%$ des arbres ont atteint le stade 4 de foliation.

\subsection{Méthodes d'estimation de la date de débourrement}

L’analyse des données est basée principalement sur les méthodes de somme de températures et de somme de coefficients de température $\left(Q_{11}\right)$. Elle a pour but d'expliquer, au moins partiellement, la diversiré des comportements observés en fonction des conditions thermiques, la variabilité génetique pouvant jouer sur la précision du modèle en se répercutant sur la confiance qu'on peut accorder aux paramètres estimés (seuil $\mathrm{S}$ ou $\mathrm{Q}_{10}$ ).

D’après les divers travaux relatifs à ce sujet, la courbe représentative de la vitesse de développement d'une plante ou d'un organe végétal en fonction de la température est de forme sigmoïde. Les ajustements proposés pour traduire de façon mathématique l'équation de la courbe sont soit linéaires (méthode de somme des températures), soit exponentiels (somme des coefficients $Q_{10}$ ). Ces mé:hodes utilisées par de très nombreux auteurs ont été analysées en détail par Durand (1967) et appliquées au débourrement du Pommier par Bidabe (1967).

Si l'on désigne par:

M la température maximale journalière,

$m$ la température minimale journalière,

$S$ le seuil thermique apparent de végétation,

les actions thermiques journalières peuvent se formaliser de la façon suivante:

- action linéaire : $a=(M-S)+(m-S)$ avec $M-S$ ou $m-S=0$ si $M$ ou $\mathrm{m} \leqslant \mathrm{S}$ (méthode des sommes de températures) ;

- action exponentielle : $a=Q_{111}{ }^{\frac{M}{111}}+Q_{111}^{\frac{m}{111}}\left(\right.$ méthodes des sommes de $\left.Q_{10}\right)$.

$M$ et $m$ sont relevées quotidiennement par la Station météorologique de Lussagnet.

Quelle que soit la méthode choisie, les actions journalières sont cumulées entre une date origine et la date d'apparition du stade phénologique étudié. Il s'avère également nécessaire de déterminer la « durée du débourrement $J$ », c'est-à-dire le nombre de jours compris entre la date d'initialisation et le stade 4 moyen de foliation. Pour un génotype donné, cette somme d'actions élémentaires doit être constante d'une année sur l'autre. Il en découle que pour optimiser les valeurs du seuil $S$ ou du $Q_{10}$ et la date origine, on fait appel à l'analyse statistique de la variabilité interannuelle des sommes d'actions journalières calculées à partir de combinaisons de différentes valeurs de ces paramètres : la meilleure combinaison est celle qui conduit à la variabilité la plus faible, c'est-à-dire au plus petit écart-type $(\sigma \mathrm{m})$ exprimé en unité du système considéré. Un calcul simple permet d'exprimer om en jours moyens de débourrement (valeur unitaire au sens de BIDABE (1967) : il devient alors possible par comparaison des écarts $2 \mathrm{om}$ de dire quelle méthode est la meilleure (mise en œuvre d'un test F). 
En ce qui concerne les deux espèces étudiées, les dates d'initialisation retenues s'échelonnent entre le $10 / 12$ et le $10 / 04$ pour le Hêtre, entre le $10 / 12$ et le $20 / 02$ pour le Chêne pédonculé. La première (10/12), choisie arbitrairement, doit être antérieure à la date de levée de dormance présumée (Galoux, 1966 ; Lavarenne-Allary, 1965 ; WAREING, 1953). Les secondes $(20 / 02$ et $10 / 04)$ dépendent de la précocité du débourrement observée. Les valeurs du seuil $\mathrm{S}$ varient de $2^{\circ}$ à $7^{\circ} \mathrm{C}$ pour le Chêne et de $-3^{\circ}$ à $7^{\circ} \mathrm{C}$ pour le Hêtre ; celles du $\mathrm{Q}_{10}$ sont comprises entre 2 et 4 pour le Chêne, entre 1,5 et 4 pour le Hêtre. Ce choix s'est effectué en fonction des valeurs trouvées pour d'autres espèces (BIDABE, 1967 ; DuRAND, 1967) pour encadrer les valeurs optimales de $S$ et de $Q_{10}$.

A chaque combinaison de la date d'initialisation et de la valeur du seuil ou du $Q_{10}$, correspond une somme d'actions thermiques, l'ensemble constituant un système. La valeur unitaire de chacun de ces systèmes, exprimée en jours moyens de débourrement, correspond au quotient de la moyenne des sommes d'actions thermiques par la durée moyenne du débourrement $\overline{\mathrm{J}}$.

L'écart $2 \sigma$ (2 écarts-types) des sommes annuelles permet d'apprécier la variation d'un système par rapport à la moyenne générale. Calculé en jours moyens de débourrement, il fournit le moyen de comparer les systèmes entre eux (BIDABE, 1967). La meilleure méthode pour estimer la date de débourrement correspond alors à celle qui présente le plus faible écart $2 \sigma\left(2 \sigma_{m}\right)$. La comparaison des écarts $2 \sigma$ à l'aide d'un test $\mathrm{F}$ permet ensuite de tester la signification statistique des différences de précision entre les systèmes étudiés.

\subsection{La défoliation}

La défoliation, chez les feuillus étudiés, est précédée de l'apparition progressive des teintes jaune, puis brun-roux sur le feuillage. La chute des feuilles s'effectue en général à partir de la teinte jaune, mais peut aussi n'affecter que des feuilles brunes ou rousses. La marcescence concerne généralement quelques individus dans un peuplement ou seulement quelques rameaux d'un ou plusieurs arbres.

Les observations hebdomadaires effectuées en 1974-1976 consistent à apprécier globalement dans un peuplement, sur $500 \mathrm{~m}^{2}$ environ, la teinte du feuillage et le taux de chute des feuilles. Une autre méthode, dérivée des travaux de Malaisse (1963) et employée en 1979 et 1980, permet d'appréhender pour le Hêtre l'ensemble du processus de défoliation. Elle consiste à distinguer dans le phénomène 5 stades caractéristiques successifs qui correspondent à 5 teintes que le feuillage prend progressivement. En ajoutant les teintes intermédiaires et le stade « 100 p. 100 de feuilles tombées », cela porte à 10 le nombre de classes (notées 1 à 10). La chute des feuilles pouvant intervenir dès la teinte jaune, l'estimation de la fraction de feuillage tombée vient alors compléter les observations sur la défoliation. Les taux de chute se répartissent selon 7 classes.

L'appréciation de la teinte et du taux de chute s'effectue pour l'ensemble de la couronne et arbre par arbre. La moyenne des observations individuelles permet ensuite d'attribuer au peuplement étudié une "teinte moyenne » et un pourcentage moyen de feuilles tombées. 
TABLEAU 2

Dates de débourrement (seuil $50 \%$ d'arbres ayant atteint le stade 4 de foliation) du Hêtre dans la chênaie-hêtraie de versant (9) et du Chêne pédonculé dans la chênaie du fond de vallée (20).

\begin{tabular}{|c|c|c|}
\hline & $\begin{array}{l}\text { Hêtre } \\
\text { P9 versant }\end{array}$ & $\begin{array}{c}\text { Chêne } \\
\text { P20, fond de vallée }\end{array}$ \\
\hline 1973 & $23 / 4$ & $20 / 4$ \\
\hline 1974 & $19 / 4$ & $2 / 4$ \\
\hline 1975 & $20 / 4$ & $7 / 4$ \\
\hline 1976 & $16 / 4$ & $8 / 4$ \\
\hline 1977 & $13 / 4$ & $22 / 3$ \\
\hline 1978 & $20 / 4$ & $5 / 4$ \\
\hline 1979 & $20 / 4$ & $12 / 4$ \\
\hline 1980 & $15 / 4$ & - \\
\hline
\end{tabular}
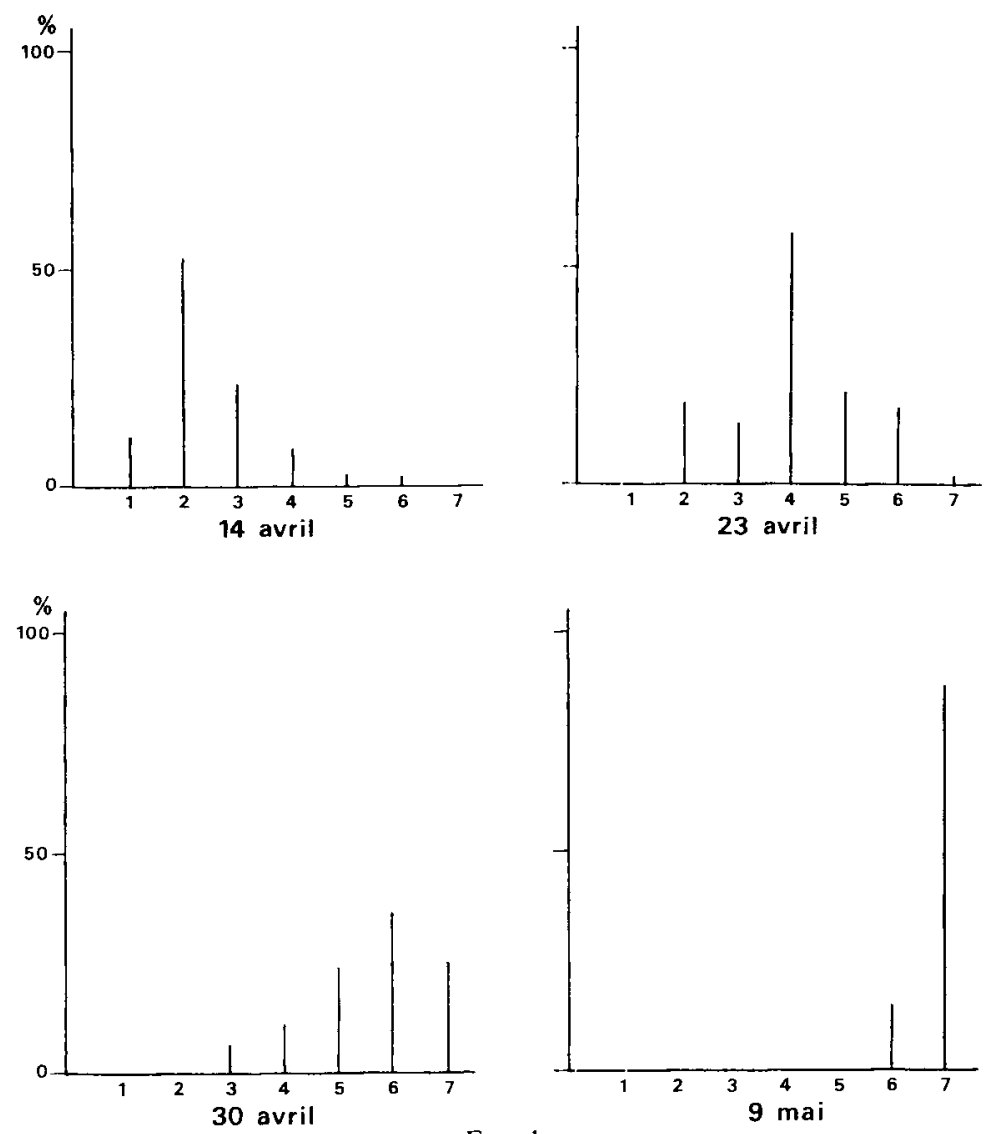

F1G. 1

Evolution de la foliation du Hêtre dans un peuplement firmé de versant (parcelle 25) en 1980 (pourcentage d'individus a chacun des 7 stades de foliation). 


\section{Résultats}

\subsection{La foliation}

\subsection{Le comportement du Hêtre}

\section{Résultat des observations}

Le tableau 2 fournit les dates de débourrement au cours de la période 1973-1980 sur la parcelle 9 où le Hêtre feuille relativement tôt. Depuis l'apparition du feuillage sur les arbres les plus précoces (seuil de $5 \%$ de débourrement moyen) jusqu'à la fin du débourrement, il s'écoule en moyenne 21 jours (de 15 à 26 jours).

A l'intérieur d'un peuplement, ce sont toujours les mêmes arbres qui manifestent le débourrement le plus précoce, quelles que soient les conditions climatiques. Pour l'ensemble d'un peuplement, l'ordre individuel de foliation n'est cependant pas rigoureusement identique d'une année à l'autre. L'écart entre les comportements extrêmes peut être très important et atteindre 30 jours pour le stade 4 . D'autre part, à un temps $\mathrm{t}$ quelconque au cours de la période de foliation, les arbres se répartissent dans les diverses classes de précocité d'une manière sensiblement gaussienne (fig. 1) ; les écartstypes ơ expriment bien l'importance de cette variabilité (tabl. 3).

TABLEAU 3

Evolution du stade moyen de foliation du Hêtre $(\bar{X})$ dans 2 peuplements de versant en 1979-1980.

\begin{tabular}{c|c|c|c|c|c|c|c|c|c}
\hline \hline \multicolumn{1}{c}{} & \multicolumn{1}{c|}{1979} & \multicolumn{4}{c}{1980} \\
\hline & & $17 / 04$ & $24 / 04$ & $2 / 05$ & $10 / 05$ & $14 / 04$ & $23 / 04$ & $30 / 04$ & $9 / 05$ \\
\hline \multirow{3}{*}{$\mathrm{P} 25$} & $\mathrm{~N}$ & 34 & 33 & 33 & 35 & 65 & 65 & 65 & 65 \\
& $\overline{\mathrm{X}}$ & 2,91 & 4,18 & 5,61 & 6,26 & 2,45 & 4,09 & 5,62 & 6,86 \\
& $\sigma$ & 0,93 & 1,24 & 0,97 & 0,61 & 1,03 & 1,39 & 1,16 & 0,35 \\
\hline \multirow{3}{*}{$\mathrm{P9}$} & $\mathrm{N}$ & 34 & 35 & 36 & 35 & 60 & 60 & 60 & 60 \\
& $\overline{\mathrm{X}}$ & 3,03 & 4,48 & 5,78 & 6,34 & 3,27 & 5,07 & 6,30 & 7,00 \\
& $\sigma$ & 1,03 & 1,25 & 0,90 & 0,54 & 1,22 & 1,07 & 0,79 & 0 \\
\hline
\end{tabular}

$\mathrm{N}=$ nombre d'individus ;

o $=$ écart-type

D'un peuplement à l'autre, les conditions stationnelles influent sur la précocité du débourrement (fig. 2). Ainsi, les hêtres situés en fond de vallée (station froide et humide) et ceux des bas de pente débourrent, en moyenne, plus tard que ceux des versants et du plateau.

Il semble, d'autre part, que les coupes d'éclaircies au sein d'un peuplement fermé accentuent la précocité du débourrement. Ainsi, les hêtres de la parcelle 9 de versant, conduite en futaie fermée jusqu'en 1978 et éclaircie à l'automne de cette même année, débourrent plus précocement en 1980 qu'au cours des années précédentes, par comparaison à d'autres parcelles de la forêt. 


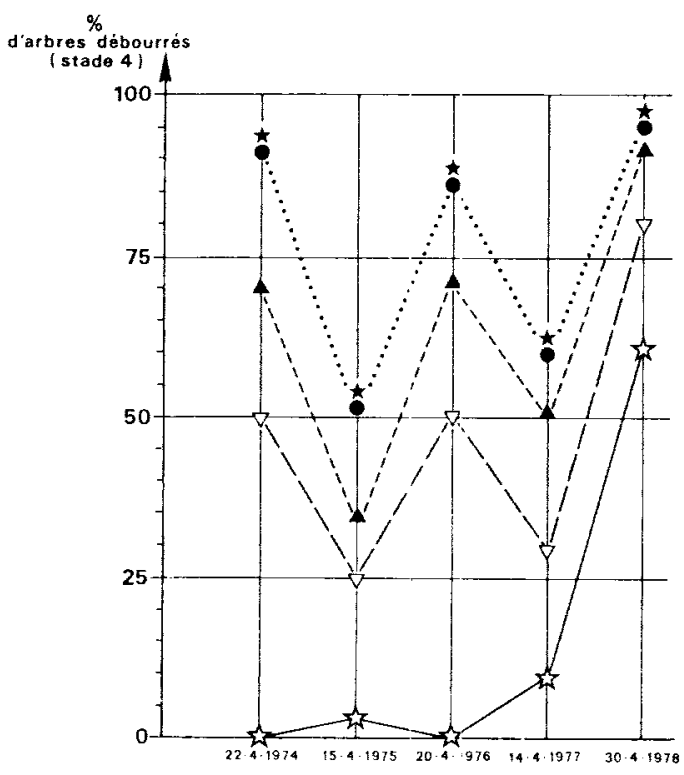

Fic. 2

Influence de la situation topographique des peuplements sur la précocité du débourrement (arbres adultes).

论 : station froide de fond de vallée (parcelle 20)

$\nabla$ : station froide de bas de versant (au-dessus de la parcelle 20)

A : vcrsant nord, au-dessus d'une coupc, peu protégé (parcelle 25)

- versant nord, protégć (parcelle 9)

$\star$ : plateau

TABLEAU 4

Analyse par le test $\chi^{2}$ des différences de dates de foliation entre les parcelles 9 et 25 . Les valeurs soulignées correspondem à des écarts significatifs au seuil de $5 \%$.

\begin{tabular}{|c|c|c|c|c|c|c|c|c|c|c|c|c|c|}
\hline \multicolumn{2}{|c|}{1974} & \multicolumn{2}{|c|}{1975} & \multicolumn{2}{|c|}{1976} & \multicolumn{2}{|c|}{1977} & \multicolumn{3}{|c|}{1979} & \multicolumn{3}{|c|}{1980} \\
\hline Dates & $x^{2}$ & Dates & $x^{2}$ & Dates & $x^{2}$ & Dates & $x^{2}$ & Dates & $x^{2}$ & $d$ & Dates & $x^{2}$ & $a$ \\
\hline $8 / 4$ & 0,26 & $7 / 4$ & 3,54 & $5 / 4$ & 0 & $22 / 3$ & 0,13 & $17 / 4$ & 1,56 & 2 & $14 / 4$ & 19.33 & 3 \\
\hline $14 / 4$ & 1,01 & $15 / 4$ & 8,62 & $12 / 4$ & 0 & $2 / 4$ & 0,07 & $24 / 4$ & 1,52 & 3 & $23 / 4$ & $\overline{20,22}$ & 3 \\
\hline $22 / 4$ & 5,06 & $21 / 4$ & $\overline{0}$ & $20 / 4$ & 2,03 & $14 / 4$ & 0.65 & $2 / 5$ & 0,43 & 2 & $30 / 4$ & 15,69 & 3 \\
\hline $29 / 4$ & $\overline{3,37}$ & $28 / 4$ & 3,37 & $30 / 4$ & 0,71 & & & $10 / 5$ & 3,00 & 1 & $9 / 5$ & 7,00 & 1 \\
\hline & & & d.d.l. & $y=1$ & & & & & & & & & \\
\hline
\end{tabular}


Pour les années 1974 à 1977, les écarts entre les deux parcelles 9 et 25 de versant ont été testés à l'aide du $\chi^{2}$ à partir de 2 classes (tabl. 4) :

- arbres ayant atteint ou dépassé le stade de débourrement (stade 4);

- arbres plus tardifs.

Au cours de ces 4 années, les écarts ne sont significatifs qu'à 2 périodes sur 15 . On peut donc admettre que les hêtres adultes ne présentent pas de différence significative quant à la précocité de la foliation d'une parcelle à l'autre.

En 1979 et 1980, les écarts sont calculés à partir des effectifs répartis dans les différentes classes de foliation. En 1979, les hêtres des deux parcelles présentent un comportement analogue; en 1980, par contre, l'écart devient très significatif aux 4 dates d'observation.

\section{Déterminisme de la date de débourrement du Hêtre (tabl. 2 et 5)}

Le tableau 5 met en évidence les écarts minimaux suivants :

- en méthode somme de températures : 2 om $=6,5$ (seuil 3 " $\mathrm{C}$ ) ;

- en méthode somme de coefficients de température : 2 om $=6,5\left(Q_{11}=2,5\right)$.

Un test $F$ permet de calculer les écarts $2 \sigma$ significativement supérieurs $(P .0,05)$ à ces valeurs minimales, soit des valeurs supérieures à 6,5 pour les deux méthodes.

TABLEAU 5

Ecarts $2 \sigma$ en jours moyens de débourrement pour les différents systèmes d'estimation de la date de foliation du Hêtre.

\begin{tabular}{|c|c|c|c|c|c|c|c|c|c|c|c|c|c|c|c|}
\hline \multicolumn{16}{|c|}{ Dates d'initialisation } \\
\hline & & $10 / 12$ & $20 / 12$ & $1 / 1$ & $10 / 1$ & $20 /$ & $1 / 2$ & $10 / 2$ & $20 / 2$ & $1 / 3$ & $10 / 3$ & $20 / 3$ & $1 / 4$ & $5 / 4$ & $10 / 4$ \\
\hline \multirow{11}{*}{$\begin{array}{c}\text { Seuil } \\
{ }^{\circ} \mathrm{C}\end{array}$} & $-3^{\circ}$ & & & 14,7 & 13,2 & 10,3 & 9,5 & 8,7 & 7,9 & 7,4 & 8,5 & 7,6 & 6,6 & 7,6 & 7,1 \\
\hline & $-2^{\circ}$ & & & 16,0 & 14,3 & 11,4 & 10,4 & 9,5 & 8,6 & 7,8 & 9,0 & 7,9 & 6,6 & 7.6 & 7,1 \\
\hline & $-1^{\prime \prime}$ & & & 17.4 & 15,5 & 12,5 & 11,5 & 10,4 & 9,3 & 8,8 & 9,5 & 8,1 & 6,6 & 7,8 & 7,2 \\
\hline & $0^{\circ}$ & & & 18,1 & 16,5 & 13,5 & 12,4 & 11,3 & 10,1 & 8,9 & 10,1 & 8,3 & 6,6 & 7,9 & 7,2 \\
\hline & $1^{\circ}$ & & & 19,6 & 17,3 & 14,4 & 14,0 & 12,3 & 11,1 & 9,6 & 10,7 & 8,6 & 6,6 & 8,0 & 7,3 \\
\hline & $2^{\circ}$ & & & 20,6 & 18,3 & 15,4 & 14,3 & 13,2 & 12,0 & 10,2 & 11,2 & 8,8 & 6,6 & 8,0 & 7,3 \\
\hline & $3^{\prime \prime}$ & 24,4 & 25,0 & 21,1 & 18,8 & 16,0 & 14,9 & 13,8 & 12,8 & 10,8 & 11,7 & 8,9 & 6,5 & 8,1 & 7.4 \\
\hline & $4^{\prime \prime}$ & 25,0 & 25,7 & 22,2 & 19,8 & 16,9 & 15,6 & 14,5 & 13,6 & 13,1 & 12,2 & 9,5 & 6,6 & 8,2 & 7,5 \\
\hline & $5^{\circ}$ & 26,3 & 27,4 & 24,0 & 21,4 & 18,1 & 16,5 & 15,4 & 14,7 & 12,4 & 12,8 & 10,1 & 6,8 & 8,4 & 7,6 \\
\hline & $6^{\circ}$ & 27,0 & 28,4 & 25,5 & 23,0 & 20,0 & 17,6 & 16,3 & 15,8 & 13,3 & 13,8 & 10,8 & 7,0 & 8,6 & 7,6 \\
\hline & $7^{\circ}$ & 27,5 & 29,6 & 27,2 & 25,0 & 20,7 & 18,8 & 18,0 & 17,0 & 14,3 & 14,4 & 11,7 & 7,3 & 8,8 & 7,7 \\
\hline $\mathbf{J}$ & & 6,4 & 6,4 & 6,4 & 6,4 & 6,4 & 6,4 & 6,4 & 6,4 & 6,9 & 6,9 & 6,9 & 6,9 & 6,9 & 6,9 \\
\hline \multirow{6}{*}{$Q_{10}$} & 1,5 & & & 6,3 & 6,0 & 4.3 & 4,3 & 4,9 & 5,2 & 5,6 & 6,6 & 6,8 & 6,6 & 7,2 & 6,9 \\
\hline & 2 & 11,0 & 11,2 & 9,4 & 8,8 & 6,6 & 6,4 & 6,2 & 6,2 & 6,3 & 7,4 & 7,2 & 6,9 & 7,7 & 6,9 \\
\hline & 2,5 & 13,2 & 14,0 & 12,4 & 11,6 & 11,4 & 8,8 & 8,2 & 8,0 & 7,5 & 8,5 & 7,9 & 6,5 & 7,7 & 6,9 \\
\hline & 3,0 & 15,2 & 16,0 & 15,0 & 16,0 & 12,2 & 11,2 & 10,0 & 9,7 & 8,8 & 11,6 & 8,7 & 6,6 & 7,0 & 7,0 \\
\hline & 3,5 & 16,8 & 18,2 & 17,4 & 16,4 & 14,4 & 13,4 & 11,6 & 11,4 & 10,2 & 10,7 & 9,5 & 6,7 & 8,2 & 7,1 \\
\hline & 4,0 & 18,4 & 20,0 & 19,6 & 18,6 & 17,2 & 15,2 & 13,4 & 12,8 & 11,5 & 11,3 & 10,2 & 6,8 & 8,4 & 7,2 \\
\hline
\end{tabular}


Tableau 6

Ecarts 2o en jours moyens de débourrement pour les différents systèmes d'estimation de la date de foliation du Chêne pédonculé.

\begin{tabular}{|c|c|c|c|c|c|c|c|c|c|}
\hline \multicolumn{10}{|c|}{ Dates d'initialisation } \\
\hline & & $10 / 12$ & $20 / 12$ & $1 / 1$ & $10 /$ & $20 / 1$ & $1 / 2$ & $10 / 2$ & $20 / 2$ \\
\hline \multirow{5}{*}{$\begin{array}{c}\text { Seuil } \\
{ }^{\circ} \mathrm{C}\end{array}$} & $3^{\circ}$ & 14,2 & 10,4 & 5,3 & $6, \angle$ & 8,6 & 12,4 & 14,6 & 17,6 \\
\hline & $4^{\circ}$ & 14,8 & 10,3 & 4,4 & 5,8 & 8,8 & 12,6 & 15,2 & 18,4 \\
\hline & $5^{\circ}$ & 14,4 & 9,2 & 3,6 & 5,5 & 9,0 & 13,0 & 15,8 & 19,4 \\
\hline & $6^{\circ}$ & 14,4 & 9.5 & 4,4 & 6,3 & 10,4 & 14,0 & 16,2 & 20,2 \\
\hline & $7^{\circ}$ & 14,0 & 10,2 & 7,5 & 8,7 & 12,6 & 15,4 & 17,6 & 21,0 \\
\hline$\overline{\mathrm{J}}$ & & 17,9 & 17,9 & 17,9 & 17,9 & 17,9 & 17,9 & 17,9 & 17,9 \\
\hline \multirow{6}{*}{$Q_{10}$} & 2 & 13,0 & 11,4 & 10,4 & 10,5 & 11,2 & 12,5 & 14,2 & 15,4 \\
\hline & 2,5 & 11,4 & 9,1 & 8,0 & 8,2 & 10,0 & 12,0 & 13,8 & 15,5 \\
\hline & 3 & 10,4 & 7,5 & 6,7 & 7,4 & 10,0 & 12,2 & 13,7 & 16,2 \\
\hline & 3,25 & 9,9 & 7,1 & 6,6 & 7,4 & 10,4 & 11,8 & 14,0 & 16,6 \\
\hline & 3,5 & 9.7 & 7,0 & 6.8 & 7,7 & 10,8 & 12,0 & 14,4 & 17, \\
\hline & 4 & 9.7 & $(7,1)$ & 7,9 & 8,8 & 12,2 & 13,6 & 15,2 & 17,1 \\
\hline
\end{tabular}

Aucune méthode d'estimation n'est vraiment supérieure à l'autre et la date optimale d'initialisation paraît être le $1^{\text {er }}$ avril. Cependant, si l'on considère le modèle exponentiel avec de faibles valeurs du $Q_{10}(2$ et 1,5$)$, les dates du 20 janvier et du $1^{\text {er }}$ février apparaissent meilleures que le $1^{\text {er }}$ avril sans toutefois que la différence soit statistiquement significative. Au $1^{\text {er }}$ avril, aucune valeur de $S$ ou de $Q_{10}$ ne manifeste une réelle supériorité sur les autres.

Pour les dates d'initialisation comprises entro le 20/12 et le 30/03 incluses, la durée moyenne du débourrement $\bar{J}$ présente un écart $2 \sigma$ inférieur, mais non significativement, aux écarts obtenus avec les autres systèmes.

\subsection{Le comportement du Chêne pédonculé (tabl. 2)}

\section{Résultat des observations}

Sur les 7 années d'observation, 2 se caractérisent par une période de débourrement plus étalée que celle des 5 autres dont la longueur moyenne est de 24 jours : 1975 (49 jours), 1977 (56 jours). En fait, au cours de ces deux années, le début de la foliation relativement précoce est suivi de périodes froides. Ainsi, entre le 22/03 et le 02/04/ 1977, le gel endommage le jeune feuillage, ce qui oblige les chênes à utiliser leurs bourgeons de remplacement. Une deuxième phase de foliation reprend alors, après un temps de latence, à partir du 22/04 pour se terminer le 09/05/1977.

Tous les ans, sans exception, le Chêne pédonculé débourre avant le Hêtre ; le décalage moyen est de 12 jours. 


\section{Déterminisme de la date de débourrement du Chêne pédonculé}

Les écarts $2 \sigma$ calculés à partir de nos 7 années d'observation sont consignés dans le tableau 6. Les écarts minimaux $2 \mathrm{om}$ sont respectivement 3,6 pour la méthode des sommes de température (avec $\mathrm{S}=5^{\circ} \mathrm{C}$ ) et 6,6 pour la méthode des sommes de $Q_{10}$ $\left(Q_{10}=3,25\right)$. Ces valeurs sont atteintes pour la même date origine de cumul des actions thermiques: le $1^{\text {or }}$ janvier.

Un test $\mathrm{F}$ permet de définir l'ensemble des écarts $2 \sigma$ significativement supérieurs (probabilité 0,05 ) à ces valeurs minimales. Ce sont les valeurs supérieures à 7,4 pour le modèle linéaire et à 13,5 pour le modèle exponentiel.

Au $1^{\text {er }}$ janvier, aucune méthode n'est en première approximation supérieure à l'autre. De plus, pour cette même date et pour chacune des deux méthodes, les valeurs optimales du seuil $\mathrm{S}$ et du $\mathrm{Q}_{10}$ ne sont pas significativement meilleures que les autres valeurs retenues pour l'analyse. Enfin, si pour le modèle somme de températures, la date du $1^{\text {er }}$ janvier semble bien la meilleure de toutes les dates choisies sauf peut-être le 10 janvier, il n'en est plus de même en système somme de $Q_{10}$ puisque du 10 décembre

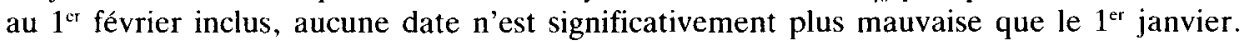

Quoiqu'il en soit, les modèles basés sur une formalisation de la seule action de la température sont plus performants pour prédire la date de débourrement du Chêne que la simple référence à la durée moyenne du débourrement $\overline{\mathrm{J}}$ pour la date optimale d'initialisation ( $1^{\mathrm{er}}$ janvier).

\subsection{La défoliation du Hêtre}

Les premières teintes jaunes apparaissent généralement au cours de la première décade d'octobre mais elles peuvent se manifester beaucoup plus tôt, dès le début septembre. Le feuillage atteint ensuite le stade «teinte jaune " au cours de la première décade de novembre et cela pour les 4 années d'observation climatiquement proches des normales d'août à octobre. A tout moment de la période de sénescence du feuillage, la répartition des arbres dans les différentes classes de pourcentage de défoliation est sensiblement gaussienne et ressemble à ce que l'on observe lors du débourrement (fig. 1 et 3 ). Les écarts-types ( $\sigma$ ) expriment l'importance de cette variabilité (tabl. 7) ; entre le premier et le dernier individus à atteindre la teinte jaune, il peut s'écouler jusqu'à 48 jours.

Le taux moyen de chute de feuilles et le stade moyen de défoliation évoluent de façon similaire. Au niveau de l'arbre, il n'existe pas de relation étroite entre le taux de chute et la teinte du feuillage.

Comme dans le cas du débourrement, il semble bien que la coupe d'éclaircie affectant la parcelle 9 de versant en 1979 ait favorisé la précocité de la défoliation du Hêtre. En effet, comme le montre le tableau 8, un seul écart entre les parcelles 9 et 25 (peuplement fermé) est significatif au seuil de $5 \%$ en 1979 (13 novembre) alors que tous les écarts deviennent significatifs en 1980. La coupe totale de la parcelle 9 en 1981 n'a pas permis la poursuite des observations et l'interprétation de nos résultats reste, de ce fait, très délicate. 

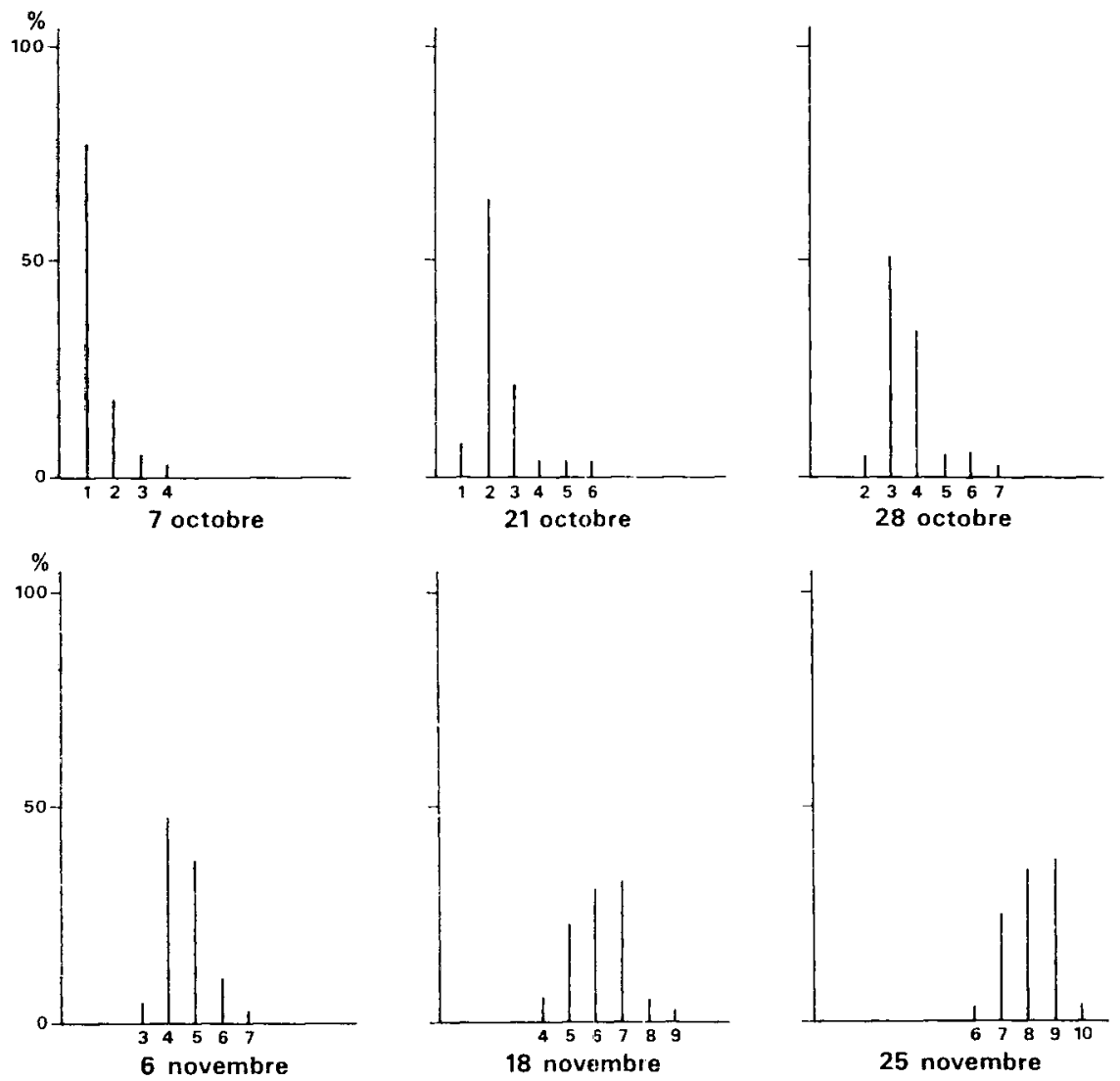

25 novembre

Fig. 3

Evolution de la défoliation du Hêtre en 1980 dans un peuplement fermé de versant (pourcentage d'individus dans chacune de: 10 classes de défoliation).

\section{TABleau 7}

Evolution du stade moyen de foliation du Hêtre $(\tilde{X})$ dans deux peuplements de versant en $1979-1980$.

\begin{tabular}{|c|c|c|c|c|c|c|c|c|c|c|c|c|c|}
\hline & & \multicolumn{6}{|c|}{1979} & \multicolumn{6}{|c|}{1980} \\
\hline & & $5 / 10$ & $16 / 10$ & $2 / 11$ & $13 / 11$ & $22 / 11$ & $30 ! 11$ & $7 / 10$ & $21 / 10$ & $28 / 10$ & $6 / 11$ & $18 / 11$ & $25 / 11$ \\
\hline P25 & $\begin{array}{l}N \\
\bar{X} \\
\sigma\end{array}$ & $\begin{array}{l}36 \\
2,03 \\
1,03\end{array}$ & $\begin{array}{l}36 \\
2,58 \\
0,91\end{array}$ & $\begin{array}{l}40 \\
4,23 \\
1,05\end{array}$ & $\begin{array}{l}40 \\
5,63 \\
0,94\end{array}$ & $\begin{array}{l}40 \\
7,13 \\
1,34\end{array}$ & $\begin{array}{l}40 \\
8.35 \\
1,21\end{array}$ & $\begin{array}{l}65 \\
1,31 \\
0,64\end{array}$ & $\begin{array}{l}65 \\
2,34 \\
0,91\end{array}$ & $\begin{array}{l}65 \\
3,50 \\
0,95\end{array}$ & $\begin{array}{l}65 \\
4,57 \\
0,79\end{array}$ & $\begin{array}{l}65 \\
6,14 \\
1,09\end{array}$ & $\begin{array}{l}65 \\
8,15 \\
0,91\end{array}$ \\
\hline P9 & $\begin{array}{l}N \\
\bar{X} \\
\sigma\end{array}$ & $\begin{array}{r}41 \\
2,37 \\
1,36\end{array}$ & $\begin{array}{l}40 \\
3,08 \\
1,07\end{array}$ & $\begin{array}{l}40 \\
4,75 \\
1,32\end{array}$ & $\begin{array}{l}37 \\
6,51 \\
1,17\end{array}$ & $\begin{array}{l}41 \\
7,44 \\
1,23\end{array}$ & $\begin{array}{l}40 \\
9,05 \\
0,96\end{array}$ & $\begin{array}{l}60 \\
1,85 \\
0,85\end{array}$ & $\begin{array}{l}60 \\
2,97 \\
1,18\end{array}$ & $\begin{array}{l}60 \\
4,25 \\
1,39\end{array}$ & $\begin{array}{l}60 \\
5,0 \\
1,18\end{array}$ & $\begin{array}{l}60 \\
6,59 \\
1,50\end{array}$ & $\begin{array}{l}60 \\
8,84 \\
0,78\end{array}$ \\
\hline
\end{tabular}

$\mathrm{N}=$ nombre d'individus ;

$\sigma=$ écart-type 


\section{Tableau 8}

Analyse par le test $\chi^{2}$ des différences de dates de défoliation entre les parcelles 9 et 25.

Dans chaque case sont mentionnées les valeurs de $\chi_{c}^{2}$ et le nombre de d.d.l.

Les valeurs soulignées correspondent à des écarts significatifs au seuil de $5 \%$.

\begin{tabular}{|c|c|c|c|c|c|c|}
\hline 1979 & $\begin{array}{l}5 \text { oct. } \\
5,22 \quad 3\end{array}$ & $\begin{array}{l}16 \text { oct. } \\
4,38 \quad 2\end{array}$ & $\begin{array}{l}2 \text { nov. } \\
6,80 \quad 3\end{array}$ & $\begin{array}{l}13 \text { nov. } \\
13,68 \quad 2\end{array}$ & $\begin{array}{l}22 \text { nov. } \\
1,43 \quad 3\end{array}$ & $\begin{array}{c}30 \text { nov. } \\
1,80 \quad 3\end{array}$ \\
\hline 1980 & $\begin{array}{c}7 \text { oct. } \\
17.43 \quad 2\end{array}$ & $\begin{array}{l}21 \text { oct. } \\
18,32 \quad 3\end{array}$ & $\begin{array}{l}28 \text { oct. } \\
12,51 \quad 2\end{array}$ & $\begin{array}{l}6 \text { nov. } \\
6,03 \quad 2\end{array}$ & $\begin{array}{l}18 \text { nov. } \\
10,85 \quad 3\end{array}$ & $\begin{array}{l}25 \text { nov. } \\
19,9 \quad 3\end{array}$ \\
\hline
\end{tabular}

\subsection{La période végétative}

Elle correspond à la phase photosynthétiquement active.

Pour le Hêtre, les auteurs fixent des limites variables à la période végétative : soit de la foliation totale jusqu'au jaunissement des feuilles (CoINTAT, 1959), soit du début de la foliation jusqu'à la coloration des feuilles (Tomescu, 1957, in Malaisse, 1964), soit encore du stade moyen 4 de foliation (débourrement) au stade moyen où les feuilles sont jaunes (Malaisse, 1964). C'est cette dernière définition qui a été adoptée en forêt de Laveyron pour les deux espèces étudiées. Ainsi comprise, la période végétative du Hêtre varie de 193 à 205 jours en fonction des années et suivant les stations ; sa durée moyenne est de 198 jours.

Cependant, des mesures réalisées sur le Noyer à Clermont-Ferrand (communication orale J.-C. MAUGET) montrent que le bilan de $\mathrm{CO}_{2}$ devient positif seulement lorsque les feuilles sont déplissées (a priori équivalent au stade 6 pour le Hêtre). Si l'on tient compte de ce résultat, la période végétative serait alors réduite d'environ 2 semaines, soit 187 jours en moyenne en 1979-1980 contre 201 selon l'évaluation précédente.

En 1980, dans le peuplement fermé, les valeurs individuelles de la période végétative varient de 171 à 221 jours, soit 50 jours d'écart avec $\sigma=10,5$ jours pour 65 individus observés. Etant donné limportance de cet écart, la corrélation entre la longueur de la période végétative et l'accroissement de diamètre mériterait d'être examinée afin de vérifier dans quelle mesure la production de bois d'un peuplement homogène dépend de la durée de la période d'assimilation.

En ce qui concerne le Chêne pédonculé en fond de vallée, la période végétative atteint, pour des années à caractéristiques climatologiques normales, la valeur moyenne de 225 jours.

\section{Discussion}

Une prévision basée sur les modèles somme de températures et somme de coefficients de température permet d'estimer l'époque de débourrement du Hêtre en forêt de Laveyron en choisissant le $1^{\text {er }}$ avril comme date d'initialisation (si l'on exclut les valeurs de $Q_{10}<2,5$ ). D'autres auteurs (Leibundgut, 1954 in Galoux, 1966...) admettent, pour d'autres régions, que la dormance est levée dès la fin de février ou même avant la fin de janvier, la foliation n'ayant lieu qu'en avril. 
Antérieurement et si l'on exclut toujours les; valeurs de $Q_{10}<2,5$, la valeur de $J$ (durée du débourrement) estime plus précisément la date de débourrement que les autres méthodes utilisées. Ces deux remarques amènent à penser que la foliation du Hêtre, plus sensible au «nombre de jours » donc à la photopériode jusqu'à la fin mars, devient ensuite plus sensible à la température à partir du $1^{\text {cr }}$ avril. C'est ainsi que le Hêtre feuille chaque année presque à la même date et le léger décalage observé correspond aux conditions climatiques particulières qui règnent dans une courte période précédant l'apparition du feuillage.

Ces résultats obtenus à partir d'observations in situ corroborent ceux de plusieurs auteurs. Wareing (1953) pense que si la levée de dormance est surtout sous la dépendance de la température dans la partie moyenne de l'aire du Hêtre (GrandeBretagne), la longueur du jour pourrait devenir le facteur déterminant dans les régions à températures printanières plus élevées (Espagne, par exemple). Des expériences de laboratoire conduites en conditions contrôlées par LAvarenne-Allary (1965), il ressort que le débourrement des bourgeons de Hêtre dépend fortement de la photopériode et ne se montre apparemment pas sensible à la température en l'absence de jours longs. Leibundgut (1954, in Galoux, 1966) observe également, en conditions contrôlées, que de jeunes hêtres soumis à des jours longs débourcent plus rapidement que les autres et il ajoute: «En avril, la longueur des jours ne paraît plus avoir d'influence sur la précocité de la mise en feuilles et c'est la hausse thermique qui déclenche le processus ». Nous ajouterons que les décalages observés d’une région à l'autre pour le début et la vitesse de la foliation dépendent vraisemblablement des facteurs thermiques locaux.

Cependant, malgré la concordance de ces divers résultats, il convient d'être prudent quant à l'interprétation du poids relatif des températures et de la photopériode et nos conclusions ne doivent servir que d'hypothèse de départ. En effet, les méthodes que nous avons employées ne sont que des ajustements plus ou moins bons à une certaine réalité dépendant de l'allure de la courbe d'action de la température sur l'évolution des bourgeons (elle-même fonction du génotype) et de l'intervalle de température dans lequel les observations sont réalisées (fonction de la station).

Les méthodes somme de températures ou somme de coefficients de température estiment plus efficacement la date de foliation du Chêne pédonculé que la durée du débourrement. Cela revient à dire, en réciproque, que le débourrement de l'espèce semble mieux réagir aux actions de la température qu'à la photopériode. Ainsi, pour les débuts de saison doux (1975 et 1977), le Chêne pédonculé commence à feuiller très tôt ; en année froide, par contre (1973), il se montre beaucoup plus tardif. Cette réponse intense à l'action de la température permet d'expliquer la plus grande précocité de son débourrement par rapport à celui du Hêtre (décalage moyen : 12 jours dans nos conditions d'observation) et cela malgré le réchauffement printanier plus lent dans le fond de vallée.

A l'inverse, dans les régions plus septentrionales, le Hêtre débourre plus tôt que le Chêne : 10 jours d'écart environ à la mi-foliation en Belgique, pour des peuplements mélangés (Galoux et al., 1967). En Creuse, Lavarenne-Allary (1965) décrit une précocité plus grande de l'une ou l'autre des deux espèces suivant les conditions climatiques du printemps : le Chêne débourre avant le Hêtre si le printemps est doux ; l'inverse se produit lorsque le printemps est froid. L'élévation plus rapide des températures dans les secteurs méridionaux de l'aire des deux espèces provoquerait le débourrement plus précoce du Chêne alors que la date à partir de laquelle l'action de la 
température sur la croissance des bourgeons de Hêtre devient prépondérante, serait la même sur l'ensemble de l'aire. Cette sensibilité du débourrement du Chêne à l'action de la température explique également une certaine vulnérabilité aux gelées tardives en région méridionale, comme ce fut le cas à Laveyron en mars 1977. Il est bien évident que ces différences régionales de comportement devraient être pondérées par la variabilité génétique des deux espèces.

Les résultats obtenus pour le Chêne confirment les observations de différents auteurs. Ainsi, Lavarenne-Allary (1965) montre que des rameaux récoltés in situ en février, débourrent en conditions contrôlées sous la seule influence de températures élevées. Prélevés entre octobre et février, les bourgeons ne se développent pas, en jours courts comme en jours longs. Seul, un traitement à l'éthylène chlorhydrine suivi de températures élevées, peut, à cette époque, permettre le débourrement. A partir de janvier-février, le Chêne acquiert donc une dormance aitionome : il se produit un changement qualitatif interne au bourgeon qui rend possible l'influence des températures élévées (dormance thermolabile ou post-dormance).

Cependant, comme dans le cas du Hêtre, il convient d'être prudent quant à l'interprétation des résultats ; chez ces deux espèces, seule une analyse de la dynamique de dormance des bourgeons en relation avec les conditions écologiques permettrait de trancher d'une manière sûre quant au poids relatif de la température et de la photopériode.

L'aptitude au débourrement précoce ou tardif chez le Hêtre apparaît, en forêt de Laveyron, comme un caractère propre à l'individu, ce qui confirme les résultats antérieurs (Schaffalitzky de Muckadell, 1959; Galoux, 1966...). En fait, entre les deux cas extrêmes, existent de nombreux intermédiaires que prouve la grande variabilité du stade de foliation entre individus à un moment déterminé (fig. 1 et tabl. 2).

L'interprétation des effets de l'éclaircie sur la précocité du débourrement est difficile, d'autant plus que les autres travaux réalisés sur le sujet n’intéressent que les jeunes hêtres (Engler, 1911 ; Helms, 1918 ; Schaffalitzky de Muckadell, 1959...). Le comportement analogue des arbres en 1979 dans les deux parcelles 9 et 25 peut s'expliquer par le fait que l'éclaircie, débutée à l'automne 1978, s'est poursuivie pendant l'hiver et n'a donc pas eu d'influence immédiate sur la précocité de la foliation. La plus grande précocité du débourrement en 1980 dans la parcelle 9 éclaircie serait, par hypothèse, liée au relèvement des températures de surface, mais les modifications du rayonnement et de la température de la saison de végétation ont pu également induire des changements de la croissance des rameaux en 1979, et, corrélativement, du repos ultérieur des bourgeons.

Le début de la sénescence du feuillage (apparition des teintes jaunes) fait vraisemblablement appel à un ensemble complexe de facteurs. La photopériode joue sans doute un rôle important (WAREING, 1953), mais la sécheresse estivale accélère le processus en réduisant l'activité physiologique des arbres : c'est ainsi que le déficit hydrique des mois d'août à septembre 1973 et 1977 a accéléré le début du jaunissement de 1 mois. Ces résultats confirment ceux de Lavarenne-Allary (1965) et de Galoux et al. (1967). De même, des mesures effectuées en 1979-1980 montrent que la période d'apparition des teintes jaunes correspond à celle où le stock d'eau du sol devient minimum (SAVOIE, 1982).

La durée de la période végétative du Hêtre en forêt de Laveyron se rapproche de celle observée en Haute-Marne par CoInTAT (1959), ce qui semble assez surprenant au 
regard des différences climatiques entre les régions considérées et le fait que sa définition de la période végétative aurait dû amplifier l'écart auquel on pouvait s'attendre; il faut cependant noter que l'auteur n'a observé qu'un nombre très faible d'individus. Nos résultats (198 jours en moyenne) s'éloignent par contre assez fortement des valeurs citées par MALAisse : 162 et 149 jours pour deux hêtraies ardennaises situées respectivement à 245 et $365 \mathrm{~m}$ d'altitude, ce qui peut s'expliquer a priori par des différences de latitude et d'altitude. Les résultats obtenus pour le Chêne vont dans le même sens : 225 jours à Laveyron en fond de vallée contre 160 jours dans une chênaie mélangée belge à $245 \mathrm{~m}$ d’altitude (Galoux et al., 1967).

Les observations sur la défoliation et la période végétative du Hêtre mettent en évidence une variation inter-individuelle importante et l'absence de corrélation, au niveau individuel, entre la précocité de défoliation et celle du débourrement. Cette observation rejoint celles de Colntat (1959) et de Malaisse (1964).

Des différences phénologiques importantes iprécocité du débourrement et de la défoliation, durée de la période végétative) sont ainsi mises en évidence selon la situation géographique des hêtraies. En 1973. Lalzi et Pignatri ont proposé un schéma illustrant les effets respectifs de la latitude et de l'altitude sur le calendrier de déroulement des principaux stades phénologiques. La forêt de Laveyron s'intègre assez bien dans ce modèle. Toutefois, à l'échelon régional, l'observation de hêtraies le long d'un transect Aquitaine-Pyrénées occidentales ne montre pas de précocité plus grande du débourrement en fonction de l'augmentation d'altitude, jusque vers $500 \mathrm{~m}$, contrairement au schéma de ces auteurs. Cette divergence provient peut-être des différences d'échelle d'observation et du fait que les points conduisant à l'établissement des courbes de LaUzi et PignatTi correspondent à des moyennes établies sur plusieurs hêtraies géographiquement distinctes et regroupées en classes de latitude et d'altitude. Enfin, un autre facteur entre-t-il peut-ètre en jeu pour explıquer les variations phénologiques des hêtraies, à savoir la variabilité génétique de l'espèce? C'est ainsi que FELBER et Thiébault (1984), Barrière et al. (1984), Comps et al. (à paraître) montrent que les hêtraies ont un polymorphisme enzymatique signnificativement plus élevé en région méridionale que dans l'aire optimale du Hêtre.

Reçu le 20 novembre 1985. Accepté le 29 septembre 1986.

\section{Summary \\ Phenology of the tree canopy in a mixed oak and beech forest in Aquitaine}

The phenologic study of the tree canopy in a mixed oak and beech forest shows seasonal behaviour of the beech tree (Fagus silvatica L.) concerming leafing out, defoliation and length of the vegetative period, close to its south-western climatic limit. Similar observations relating io Quercus pedunculata allowed us to compare the phenoligy of both species, one with the other in Aquitaine, and with that of populations further north in the optimal area of the beccli.

In Aquitaine, leafing out-grade 4 time can be estimated - using temperatures sum methods - by choosing the beginning of April as "initialisation time ". In this latitude, the higher spring temperatures favour leafing out precocity and speed. A discussion is initiated in order to estimate the respective influence of photoperiod and temperature upon foliation time. 
Leafing out and defoliation precocity of beech tree are individual characters; thus, they most likely depend on its genotype.

Oak-tree leafing out seems to be more sensitive to temperature than to photoperiod and it is possible to estimate leafing out-grade 4 time by choosing January 1st as "initialisation time ". Contrary to the behaviour of both species in the regions further north, the beech-tree leafs out later than the oak-tree.

Key words : Leafing out, vegetative period, temperatures sum, beech-tree, oak-tree (Quercus pedunculata), Aquitaine.

\section{Références bibliographiques}

Barrière G., Comps B., Cuguen J., N'Tsiba F., Thiébaut B., 1985. The genetical ecological variability of Beech (Fagus silvatica L.) in Europe. An alloenzymatic study : genetic isolations of beechwoods. Proceed. of the first Symposium "Improvement and Silviculture of Beech", Grosshansdorf (R.F.A.), May 31 - June 4, 1984, IUFRO Project Groupe P1 10-00, $24-50$.

Baumgartner A., Kleinlein G. und Waldmann G., 1959. Forstlichphänologische Beobachtungen und Experimente am Grossen Falkenstein (Bayerischer Wald). Forstwissenschaft. Centralblatt., 75 Jhrg., 290-303.

Becker M., 1981. La phénologie des hêtraies. Le Hêtre, INRA Ed., 108-117.

Bidabe B., 1967. Action de la température sur l'évolution des bourgeons de Pommier et comparaison de méthodes de contrôle de l'époque de floraison. Ann. Physiol. vég., 9 (1), 6586.

Cointat M., 1959. Observations sur la foliation du Hêtre. Rev. forest. franç., 3, 214-217.

Comps B., Barrière G., Merzeau D., Letouzey J., (à paraître). La variabilité alloenzymatique des hêtraies dans les sous-domaines médio- et eu-atlantiques. Can. J. Forest Res.

Comps B. Letouzey J., Timbal J., 1980. Essai de synthèse phytosociologique sur les hêtraies collinéennes du domaine atlantique français. II - Les hêtraies sur sols acides et neutres. Doc. phytosociol., N.S., Lille, IV, 177-191.

Durand R., 1967. Action de la température et du rayonnement sur la croissance. Ann. Physiol. vég., $9(1), 5-27$.

ENGLER A., 1911. Untersuchungen über den Blattansbruch und das sonstige Verhalten von Schatten- und Lichtpflanzen der Buche und einiger anderer Laubhölzer. Mitt. schweiz. Anst. forstl. Versuchw., 10, 105-188.

Felber F., Thiébaut B., 1984. Etude préliminaire sur le polymorphisme enzymatique du hêtre, Fagus silvatica L. - Variabilité génétique de deux systèmes de peroxydases en relation avec les conditions écologiques. Acta Oecologica, Oecol. Plant., 5 (19), $\mathrm{n}^{\circ} 2,133-150$.

Galoux A., 1966. La variabilité génécologique du hêtre commun (Fagus silvatica L.) en Belgique. Station Rech. Eaux et Foréts Groenendaal-Hoeilaart, Travaux sér. A, 11, 122 p.

Galoux A., Scinock G., Grulois J., 1967. Recherches sur l'écosystème forêt. Série B : La chênaie mélangée calcicole de Virelles-Blaimont. Contribution $\mathbf{N}^{\circ} 5$ : la variabilité phéno:Đgique et les conditions climatiques. Bull. Soc. r. Bot. Belgique, 100 (2), 309-314.

Helms L.A., 1918. Lysets Indvirkning pa Bogens Lovspring Dansk Skovforen. Tidsskr., 3, 520535 .

Krahl-Urban J., 1958. Vorlaüfige Ergebnisse von Buchen-Provenienzversuchen. Allg. Forst- und Jagdzeitung, $129 \mathrm{Jhrg}, 242-243$.

Lausi D., Pignattı S., 1973. Die Phänologie der europäischen Buchenwälder auf Pflanzensoziologischer Grundlage. Phytocoenologia, 1 (1), 1-63.

Lavarenne-Allary S., 1965. Recherches sur la croissance des bourgeons de Chêne et de quelques autres espèces ligneuses. Ann. Sci. For., Nancy, 22, 1-210. 
Leibundgut H. et Kunz R., 1952. Zur Phänologie der Laubbaüme, insbesondere der Buche. Schweizerische Zeitschrift für Forstwesen, 29-45

Malaisse F., 1963. Contribution à l'étude des hêtraies d'Europe occidentale. Note 3. Remarques préliminaires sur la phénologie des hêtraies. Les Naturalistes Belges, 44 (8), 369-383.

Malaisse F., 1964. Contribution à l'étude des hêtraics de l'Europe occidentale. Note 4. Quelques observations phénologiques de hêtraies en 1962. Bull. Soc. r. Bot. Belgique, 97, 85-87.

Riedacker A., 1981. Physiologie de la croissance du Hêtre - Croissance aérienne et souterraine. Le Hêtre, INRA Ed., 160-169.

SAvoıe J.-M., 1982. Une chênaie-hêtraie d'Aquitaine : la forêt de Laveryon (Landes). Facteurs stationnels, phénologie, régénération du hêtre. Thèse Doct. $3^{e}$ cycle, Université de Bordeaux I, 109 p., 72 fig., 21 tabl.

Schaffalmzky de Muckadell M., 1959. Investigations on aging of apical meristems in woody plants and its importance in silviculture. Dit. Forstlige Forsogsvaesen, Danmarck, XXV, 310447.

WAREING P.F., 1953. Growth studies in woody species. V - Photoperiudism in dormant buds of Fagus silvatica L. Physiol. Plant., 6, 692-706. 